\title{
Quasi-phasematching in a poled Josephson traveling-wave parametric amplifier with three-wave mixing
}

\author{
A. B. Zorin * \\ Physikalisch-Technische Bundesanstalt, Bundesallee 100, 38116 Braunschweig, Germany
}

(Dated: May 15, 2021)

\begin{abstract}
We develop the concept of quasi-phasematching (QPM) by implementing it in the recently proposed Josephson traveling-wave parametric amplifier (JTWPA) with three-wave mixing (3WM). The amplifier is based on a ladder transmission line consisting of flux-biased radio-frequency SQUIDs whose nonlinearity is of $\chi^{(2)}$-type. QPM is achieved in the $3 \mathrm{WM}$ process, $\omega_{p}=\omega_{s}+\omega_{i}$ (where $\omega_{p}$, $\omega_{s}$, and $\omega_{i}$ are the pump, signal, and idler frequencies, respectively) due to designing the JTWPA to include periodically inverted groups of these SQUIDs that reverse the sign of the nonlinearity. Modeling shows that the JTWPA bandwidth is relatively large (ca. $0.4 \omega_{p}$ ) and flat, while unwanted modes, including $\omega_{2 p}=2 \omega_{p}, \omega_{+}=\omega_{p}+\omega_{s}, \omega_{-}=2 \omega_{p}-\omega_{s}$, etc., are strongly suppressed with the help of engineered dispersion.
\end{abstract}

Due to vanishingly small losses and an ultimately quantum level of internal noise, $\stackrel{1}{\stackrel{1}{b}}$ cryogenic travelingmicrowave parametric amplifiers based on the kinetic inductance of superconducting wires ${ }^{2}-\underline{4}$ and Josephson junctions $s^{\underline{5-8}}$ are considered highly useful quantum devices that can be applied in precision quantum measurements, photon detection, quantum communication and quantum computing, ${ }_{1}^{9,10}$ In comparison to their cavity-based counterparts,, $11-13$ traveling-wave parametric amplifiers have the advantages of a wider bandwidth and a larger dynamic range. However, realization of phase matching that is sufficient for ensuring large (ideally, exponential $\frac{14}{4}$ ) flat signal gains in a wide bandwidth remains the greatest challenge when designing such amplifiers,$\underline{\underline{5}-\underline{8} \cdot 15-17}$

Josephson traveling-wave parametric amplifiers (JTWPAs) have the architecture of a transmission line with discrete elements $\underline{18}$ Their performance is normally based on a Kerr-like nonlinearity, i.e., a Josephson relation between a small current $I$ and a small flux $\Phi$ of the form $I=\left(\Phi-\gamma \varphi_{0}^{-2} \Phi^{3}\right) L_{0}^{-1}$, where $L_{0}^{-1}$ is the inverse inductance, $\varphi_{0}=\Phi_{0} / 2 \pi=\hbar / 2 e$ is normalized flux quantum, and $\gamma(>0)$ is the Kerr coefficient $\underline{19}$ These amplifiers are operated in a four-wave mixing (4WM) regime wherein the pump $\left(\omega_{p}\right)$, signal $\left(\omega_{s}\right)$, and idler $\left(\omega_{i}\right)$ frequencies obey the relation $2 \omega_{p}-\omega_{s}-\omega_{i}=0$. However, here, it is difficult to fulfill the phase-matching relation for corresponding wavenumbers, $\Delta k=2 k_{p}-k_{s}-k_{i}=0$, in a wide range of frequencies and pump powers. Specifically, the Kerr effect causes unwanted self-phase modulation (SPM) and cross-phase modulation (XPM) of waves because of the intensity-dependent phase velocities leading to phase shifts $\underline{14}$ This leads to imperfect phase matching, $\Delta k \neq 0$, which can only be improved by means of dispersion engineering. This can be done, for example,

This article may be downloaded for personal use only. Any other use requires prior permission of the author and AIP Publishing. This article appeared in Appl. Phys. Lett. 118, 222601 (2021) and may be found at https://doi.org/10.1063/5.0050787 by applying resonant phase matching $5,6,15$ or by inverting the Kerr coefficient sign, $\gamma<0 \underline{\underline{8} .16}$

Recently, superconducting elements that possess widely tunable non-centrosymmetric nonlinearity of the $\chi^{(2)}$-type have been proposed. Such elements (see Fig. 1) include either non-hysteretic radio-frequency SQUIDs $\left(\right.$ rf-SQUIDs) ${ }^{20}$ or asymmetric multijunction SQUIDs, i.e., so-called superconducting nonlinear asymmetric inductive elements (SNAILs) $\stackrel{21,22}{=}$ An appropriate magnetic flux $\Phi_{e}$ applied to these SQUIDs allows the currentflux relation to be adjusted to fit the form

$$
I=\left(\Phi-\beta \varphi_{0}^{-1} \Phi^{2}\right) L_{0}^{-1},
$$

where coefficient $\beta$ is the electrical analog of the susceptibility tensor $\chi^{(2)}$ in the optics $\underline{\underline{14}}$ Formula (11) provides the means for designing Kerr-free $(\gamma=0)$ JTWPAs with pure three-wave mixing ( $3 \mathrm{WM}), \omega_{p}-\omega_{s}-\omega_{i}=0, \underline{20,22,23}$ Here, the pump frequency $\omega_{p}$ favorably lies outside the signal band, $\left(0, \omega_{p}\right)$.

Another remarkable property of $3 \mathrm{WM}$ is the absence of SPM and XPM effects. Hence, for a sufficiently small dispersion, the phase-matching condition, $k_{p}-k_{s}-k_{i} \approx 0$, can be met in a wide frequency range. The dispersion relation in the ladder-type transmission line is $\underline{\underline{24}}$

$k=2 \arcsin \left(\frac{\omega / 2 \omega_{0}}{\sqrt{1-\omega^{2} / \omega_{J}^{2}}}\right) \approx \frac{\omega}{\omega_{0}}\left(1+\frac{\omega^{2}}{2 \omega_{J}^{2}}+\frac{\omega^{2}}{24 \omega_{0}^{2}}\right)$,

where $k$ is the wavenumber normalized on the reverse size of elementary cell $d^{-1}$ and the frequencies $\omega_{J}=1 / \sqrt{L C_{J}}$ and $\omega_{0}=1 / \sqrt{L C_{0}}$ (see notations in Fig. 1) are the SQUID plasma frequency and the line cutoff frequency, respectively. Thus, the condition of small dispersion reads $\omega_{p} \ll \omega_{J}, \omega_{0}$.

In general, mixing of the signal and the pump using the nonlinearity given by Eq. (10) results not only in the idler wave being generated with difference frequency $\omega_{i}=$ $\omega_{p}-\omega_{s}<\omega_{p}$ but also in waves with frequencies above $\omega_{p}$, including $\omega_{+}=\omega_{p}+\omega_{s}, \omega_{-}=2 \omega_{p}-\omega_{s}=\omega_{p}+\omega_{i}$, $2 \omega_{p}$. Moreover, for vanishingly small dispersion, phasematching conditions for these accompanying processes 
(a)

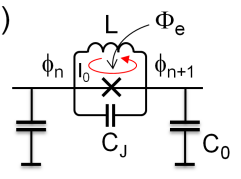

(b)

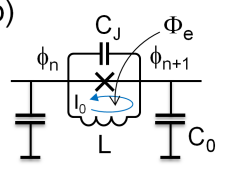

(c)

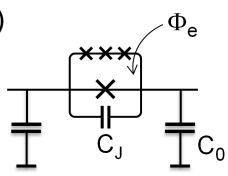

FIG. 1: Fragments of superconducting transmission lines with nonlinearities of the $\chi^{(2)}$-type due to flux-biased rf-SQUIDs (Josephson junctions are denoted by crosses). Panels (a) and (b) show inverted configurations of rf-SQUIDs, which have opposite signs of coefficient $\beta$ in a uniform external magnetic field. In both cases, (a) and (b), the circulating current $I_{0}$ is anticlockwise. The screening parameter value in these rfSQUIDs, $\beta_{L}=L I_{c} / \varphi_{0}<1$, where $I_{c}$ is the Josephson critical current ${ }^{20}$ (c) SNAIL circuit in which a short array of Josephson junctions that forms the upper SQUID branch serves as the equivalent (kinetic) inductor $L:^{21,22}$

may also be met. In the original concept of the parametric amplifier with $3 \mathrm{WM}, \underline{25}, \underline{26}$ these waves were excluded, so their effects on the basic waves were not considered. However, later analysis showed $24,27,28$ that these modes not only absorb pump energy (in accordance with the Manley-Rowe relations ${ }^{29}$ ), but also interact with signal and idler waves, causing notable signal-rise undulation. 24 As a result, premature pump depletion and irregular dependence of the gain on the signal frequency and pump power were observed in both the simulations ${ }^{28}$ and the experiment $\underline{22,23}$

A straightforward method of suppressing the propagation of unwanted high-frequency modes is to reduce the cutoff frequency $\omega_{0}$ in such a way that $\omega_{p} \lesssim \omega_{0}<$ $\omega_{ \pm}, \omega_{2 p}$. However, this approach has several drawbacks: First, because the wavelength $\lambda=2 \pi \omega_{0} / \omega$ is only about the size of 10 cells, the discreteness of the line becomes critical; thus, its transmission and, hence, the gain may exhibit notable ripples. Second, in order to keep the impedance unchanged, i.e., $Z_{0}=\sqrt{L / C_{0}}=50 \Omega$, both the inductance $L$ and the ground capacitance $C_{0}$ should be increased; this is possible only by increasing their physical sizes, which may entail difficulties in fabricating the circuit on a standard chip. Third, the maximum pump power is $P_{p}^{\max } \approx\left(\omega_{0} \varphi_{0}\right)^{2} / 2 Z_{0} \stackrel{20}{\stackrel{20}{\rightleftarrows}}$ thus, a small value of $\omega_{0}$ will yield a small $P_{p}^{\max }$ and, hence, cause pump depletion at a rather low signal power.

To solve this problem, we first engineered a reasonably large dispersion. By setting the SQUID plasma frequency slightly above the double pump frequency, $\omega_{J} \gtrsim 2 \omega_{p}$, which is done by increasing the SQUID capacitance $C_{J}$, a phase mismatch for unwanted modes, $\omega_{+}, \omega_{-}$, and $\omega_{2 p}$ (all of which are between $\omega_{p}$ and $2 \omega_{p}$ ) can be made sufficiently large in accordance with formula (21). The unavoidable phase mismatch for basic 3WM, $\Delta k_{0}=k_{p}-k_{s}-k_{i}>0$, is not dramatically large in this case, although it depends on the signal frequency $\omega_{s} \stackrel{\underline{24}}{=}$ For example, for $\omega_{s} \approx \omega_{i} \approx 0.5 \omega_{p}$, the mismatch is at its maximum, $\Delta k_{0} \approx 3 \omega_{p}^{3} / 8 \omega_{J}^{2} \omega_{0} \sim 3 \omega_{p} / 32 \omega_{0}$. This

value corresponds to a reasonably large coherence length of $\ell_{c}=\pi / \Delta k_{0} \sim 10^{2}$ cells. In principle, this mismatch can be improved by applying either resonator phasematching $5.6,15$ ? or periodic loading of the line, $, 2,4,27$

In this Letter, however, we use so-called quasiphasematching (QPM) $\stackrel{30,31}{=}$ whose possible implementation in our JTWPA is outlined in Fig. 2. QPM was originally exploited in optical devices, including parametric amplifiers, 32 parametric oscillators,$\frac{33}{2}$ secondharmonic generators,$\stackrel{34.35}{,}$ and spontaneous parametric down-converters $\stackrel{36}{\underline{3}}$ These devices were based on $\chi^{(2)}$ nonlinearity in a poled optical material like $\mathrm{LiNbO}_{3}$.

The core operating principles of QPM are as follows. After passing coherence length $\ell_{c}$ each time the signal wave is about to begin to decrease (as a consequence of the wavenumber mismatch), a reversal of the $\chi^{(2)}$-sign occurs, which changes the signal phase by $\pi$ and allows the wave amplitude to continue to grow. When another $\pi$ phase shift has accumulated, the constant-sign domain is reversed again, and so on. Thus, due to the periodic inversion of the orientation (poling) of the $\mathrm{LiNbO}_{3}$ crystal, an effective compensation of the phase mismatch $\Delta k_{0}$ in $3 \mathrm{WM}$ is possible $\underline{\underline{31}}$

The mechanism of QPM can be illustrated using simplified coupled-mode equations (CMEs) $\stackrel{14}{\underline{14}}$ for slowly varying complex amplitudes of the signal and idler waves, $A_{s}$ and $A_{i}$, respectively, which are coupled to the pump wave with amplitude $A_{p}$. The corresponding signal

(a)

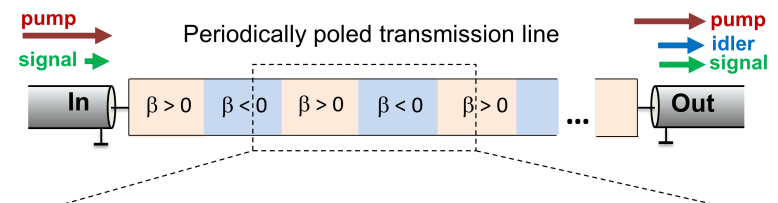

(b)

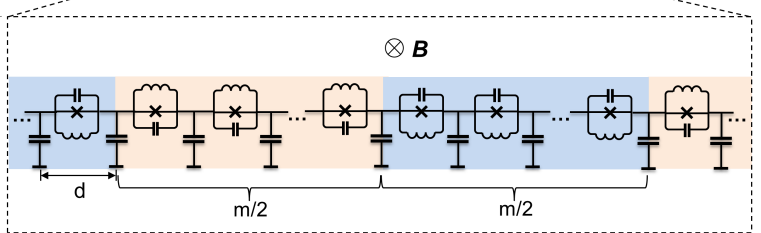

(c)

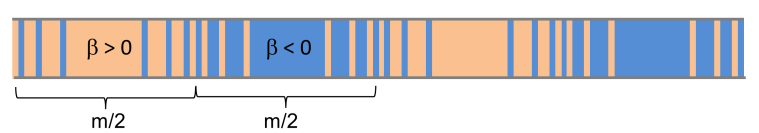

FIG. 2: (a) Schematic circuit diagram of a QPM-based JTWPA comprising a serial array of $N\left(\sim 10^{3}\right)$ flux-biased rfSQUIDs with a periodically inverted sign of nonlinear coefficient $\beta$. (b) Poled segments of the line that have different signs of coefficient $\beta=|\beta| \operatorname{sgn}[\sin (2 \pi n / m)]$, which are achieved due to the inversion of rf-SQUIDs in a uniform magnetic field $\boldsymbol{B}$. (c) A pulse-width modulated profile that synthesizes a smooth variation of the effective local value of coefficient $\beta(x)$; the different segments, which have opposite signs of $\beta$, are shown by means of different colors. 
(idler) wave is given by the expression $\phi_{s, i}(x, t)=$ $0.5 A_{s, i}(x) e^{i\left(k_{s, i} x-\omega_{s, i} t\right)}+$ c.c., where $x$ is a continuous coordinate that is normalized on cell size $d$. Thus, the superconductor phase $\phi_{s, i}(x, t)$ on the $n$-th node at $x=n$ takes the value $\phi_{n}^{(s, i)}(t)=\Phi_{n}^{(s, i)}(t) / \varphi_{0} \stackrel{20}{\underline{20}}$ Treating the pump as undepleted, $\left|A_{p}(x)\right|=A_{p 0} \gg\left|A_{s, i}(x)\right|$, the CMEs for Kerr-free $(\gamma=0) 3 \mathrm{WM}$ read

$$
\begin{aligned}
& \frac{d A_{s}}{d x}=-0.5 \eta(x)|\beta| k_{p} k_{i} A_{p 0} A_{i}^{*}(x) e^{i \Delta k_{0} x} \\
& \frac{d A_{i}}{d x}=-0.5 \eta(x)|\beta| k_{p} k_{s} A_{p 0} A_{s}^{*}(x) e^{i \Delta k_{0} x} .
\end{aligned}
$$

The peculiarity of these otherwise standard ${ }^{14}$ equations is the presence of modulation term $\eta(x)$, a periodic function with a period of $x_{m}=m$. This function takes only binary values, i.e., 1 or -1 , and thus determines the sign of $\beta$. To capture the QPM effect, we approximate the function $\eta(x)$ by the dominant (first) term in its Fourier series expansion, 33 i.e., $\eta(x) \approx \eta_{0} \sin k_{m} x$. Then, the exponent entered on the right-hand side of Eqs. (314), takes the form

$$
\eta(x) e^{i \Delta k_{0} x}=\frac{\eta_{0}}{2 i}\left[e^{i\left(\Delta k_{0}+k_{m}\right) x}-e^{i\left(\Delta k_{0}-k_{m}\right) x}\right] .
$$

Choosing a poling period $m$ in such a way that $k_{m} \equiv$ $2 \pi / m=\Delta k_{0}$, i.e., the actual QPM condition, the first (fast oscillating) term on the right-hand side of Eq. (5) can be omitted as its integral effect is apparently small, while the second term, $e^{i\left(\Delta k_{0}-k_{m}\right) x}=1$, enables efficient parametric mixing. As a result, an exponentially increasing solution, $\left|A_{s, i}\right| \propto e^{g x}$, in which the exponential gain factor is $g=0.25 k_{p} \sqrt{k_{s} k_{i}} \beta_{\text {eff }} A_{p 0}$, takes place on a much larger scale than $m=2 \pi / k_{m}=2 \ell_{c}$ 는 The effective value of the nonlinear coefficient is $\beta_{\text {eff }}=0.5 \eta_{0}|\beta|$. If the function $\eta(x)$ has a meander shape with a $50 \%$ duty cycle, the Fourier coefficient is $\eta_{0}=4 / \pi$, yielding $\beta_{\text {eff }}=(2 / \pi)|\beta|<|\beta|$, 年 The resulting reduction in gain can be compensated by applying a somewhat larger pump amplitude $A_{p 0}$ and/or an increasing length $N$.

In contrast to the optical-material QPM technologies $\stackrel{30,33,36}{=}$ the rf-SQUID-based transmission line can be poled in a relatively simple way. A perpendicular magnetic field $\boldsymbol{B}$ creates a magnetic flux $\Phi_{e}=|\boldsymbol{B}| S$ that is applied to each SQUID; here, $S$ is the loop area. Assuming without loss of generality that $0<\Phi_{e} \leq 0.5 \Phi_{0}$, one obtains an anticlockwise direction for the circulating current $I_{0}$ independently of the SQUID configuration (as shown in Figs. 1(a) and 1(b)). Then, defining the constant phase drop on the Josephson junction as $\phi_{\mathrm{dc}}=\phi_{n}-\phi_{n+1}$, we arrive at an rf-SQUID equation for the magnetic flux ${ }^{37}$ in the form

$$
\Phi_{e} / \varphi_{0}= \pm \phi_{\mathrm{dc}} \pm \beta_{L} \sin \phi_{\mathrm{dc}},
$$

where the sign $+(-)$ corresponds to the configuration shown in Fig. 1(a) (1(b)). A change in the sign of the right-hand side of this equation is equivalent to a change of $\Phi_{e} \rightarrow-\Phi_{e}$ (or, equivalently, a virtual flip of the magnetic field $\boldsymbol{B}$ ). As a result, the optimal phase drop, $\phi_{\mathrm{dc}}=\pi / 2$, which ensures both a zero Kerr coefficient $\gamma$ (even periodic function of flux $\Phi_{e}$ ) and nearly the maximum of coefficient $\beta$ (odd periodic function of $\Phi_{e}$ ), changes its value, $\pi / 2 \rightarrow-\pi / 2$ (see Fig. 3 ). Thus, the sign of nonlinearity constant $\beta$ is flipped, $\beta \rightarrow-\beta$.

To demonstrate the efficiency of the QPM concept for JTWPA with $3 \mathrm{WM}$, we modeled the circuit with the architecture shown in Fig. 2, neglecting possible small losses. We designed a sufficiently low plasma frequency $\omega_{J}$, i.e., $2 \omega_{p} \lesssim \omega_{J} \ll \omega_{0}$. For the analysis of this circuit, we used the set of six $\mathrm{CMEs}^{38}$ (the so-called CME-2 set ${ }^{28}$ ), which takes into consideration all significant modes with frequencies below the actual transmission threshold of $\omega_{J}$, i.e., $\omega_{s}, \omega_{i}, \omega_{p}, \omega_{+}, \omega_{-}$, and $\omega_{2 p}$, and thus describes all relevant mixing processes involving large pump. The set of these equations was numerically solved 38 by means of the standard Runge-Kutta method. The modeling showed the maximum cross-gain for modes $\omega_{+}$and $\omega_{-}$was safely smaller than the direct signal gain. The pump power converted into the second harmonic, $\omega_{2 p}$, was much less than $1 \%$.

Figure 4(a) shows three types of nonlinearity profiles $\eta(x)=\beta(x) / \beta_{\max }$ that have similar periods $m$. This set includes (i) a meander with a $50 \%$ duty cycle, i.e., $\eta(x)=$ $\operatorname{sgn}[\sin (2 \pi x / m)]$ (violet curve); (ii) a tapered meander (widely used in optics for reducing the gain ripple ${ }^{32}$ ), whose positive half-period $(0 \leq x \leq m / 2)$ is described in our case by the formula

$$
\eta(x)=-a \ln \left[e^{-2 x / a b m}+e^{-1 / a}+e^{-(m-2 x) / a b m}\right]
$$

with fixed parameter values, $a=0.15$ and $b=0.2($ red curve); and (iii) a sinusoidal profile, $\eta(x)=\sin (2 \pi x / m)$

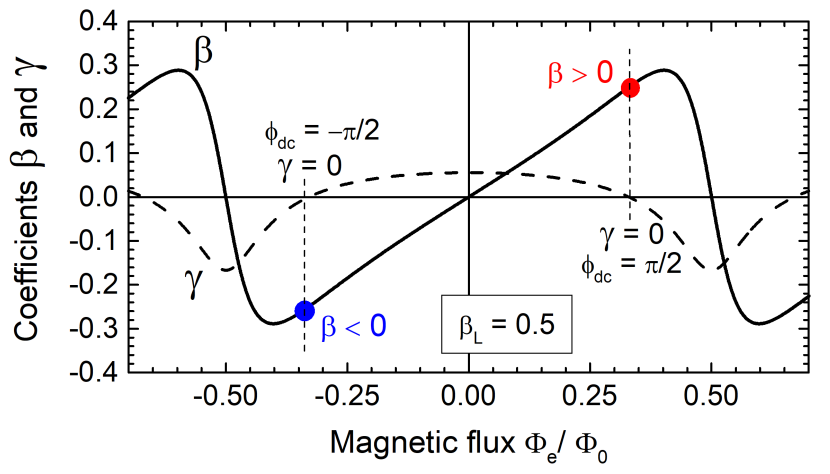

FIG. 3: Nonlinear coefficients $\beta$ (solid curve) and $\gamma$ (dashed curve) versus external magnetic flux $\Phi_{e}$. The red and blue solid circles indicate the optimal working points which correspond to $\phi_{\mathrm{dc}}= \pm \pi / 2$ (yielding zero Kerr coefficient, $\gamma=0$ ) for rf-SQUID configurations shown in Figs. 1(a) and 1(b), respectively. Note that the inductance of the Josephson junction at these points is infinite, while the total inductance of the rf-SQUID is given by the linear inductance $L$, $L_{0}=L /\left(1+\beta_{L} \cos \phi_{\mathrm{dc}}\right)=L^{22}$ 
(green curve). To implement shapes (ii) and (iii), they were "digitized" using pulse-width modulation, 38 resulting in a variable density of $\beta$-flips per unit length. ${ }^{39}$ The transmission line poled in this fashion is schematically shown in Fig. 2(c).

The signal gain in JTWPA with $N=1000$ and $\beta_{L}=0.5$, which is calculated for poling profiles (i)(iii), is shown in Fig. प(b) as a function of frequency. The pump frequency and the line characteristic frequencies were chosen in such a way that $\omega_{p} / \omega_{J} \approx 0.43$ and $\omega_{p} / \omega_{0} \approx 0.17$, which yielded a relatively large chromatic dispersion (see Eq. (21) and thus a sufficiently large phase mismatch for unwanted high-frequency modes, $\omega_{ \pm}$and $\omega_{2 p}$. As a result, the coherence lengths for these waves, assuming $\omega_{s} \approx \omega_{i} \approx 0.5 \omega_{p}$, were $\ell_{+}=\ell_{-}=54$ and

(a)

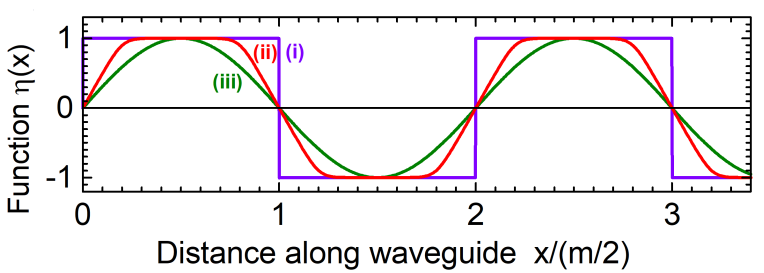

(b)

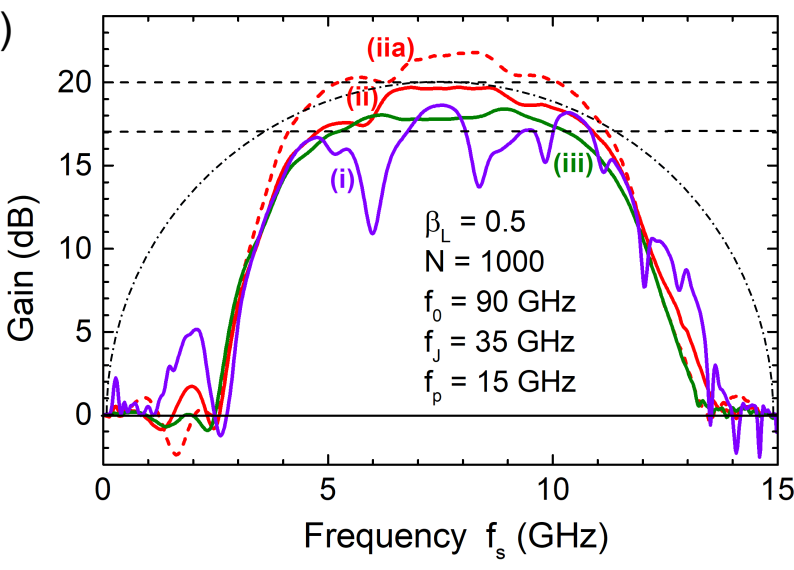

FIG. 4: (a) Three types of periodic poling shapes $\eta(x) / \eta_{0}$ that enable QPM: (i) meander with a $50 \%$ duty cycle (violet curve); (ii) tapered meander (red curve); and (iii) sinusoidal shape (green curve). (b) Gain versus signal frequency in quasi-phasematched JTWPAs that have periodic poling of three types, (i)-(iii). The curves have the same colors as in panel (a). The circuit parameters for the given frequencies $\omega_{0}, \omega_{J}$, and $\omega_{p}$, the screening parameter $\beta_{L}$, and the impedance $Z_{0}$ of $50 \Omega$ are as follows: $L=Z_{0} / \omega_{0} \approx 88 \mathrm{pH}$, $C_{0}=1 / \omega_{0} Z_{0} \approx 32 \mathrm{fF}, C_{J}=1 / \omega_{J}^{2} L \approx 235 \mathrm{fF}, I_{c}=\beta_{L} \varphi_{0} / L \approx$ $1.8 \mu \mathrm{A},|\beta|=0.24, m=448$ for solid curves (i), (ii), and (iii) and $m=420$ for dashed curve (iia). The undepleted pump power (ca. $-70 \mathrm{dBm})$ ensures that the phase oscillations on every rf-SQUID have an amplitude of $\phi_{p}=\left(\omega_{p} / \omega_{0}\right) A_{p 0} \approx 0.5$ rad. The dash-doted semiellipse shows the shape of the signal gain in an ideal $3 \mathrm{WM}$ parametric amplifier that has perfect phase matching in the full range $\left(0, \omega_{p}\right)$ and thus a wider bandwidth ${ }^{26}$ $\ell_{2 p}=11$, respectively. (By comparison, the pump wavelength was $\lambda_{p}=2 \pi / k_{p} \approx 2 \pi \omega_{0} / \omega_{p}=38$.) The phase mismatch for the basic $3 \mathrm{WM}$ process was found to be $\Delta k_{0} \approx 0.014$, which yielded a sufficiently large coherence length, $\ell_{c}=\pi / \Delta k_{0}=224 \gg \ell_{ \pm}, \ell_{2 p}$. Thus, the designed value of the poling period, $m=2 \ell_{c}$, amounted to 448 .

The maximum gain of $20 \mathrm{~dB}$, which was achieved for QPM profile of type (ii) at $\omega_{s} \sim 0.5 \omega_{p}$, only roughly corresponds to the theoretical value for QPM gain, whose formula is valid for $N / m \gg 1$. Taking into account the effective value of the nonlinear coefficient, $\beta_{\mathrm{eff}}=2|\beta| / \pi \approx$ 0.16 , the exponential gain factor is $\underline{20}$

$$
g=0.25 \eta_{\mathrm{eff}} \phi_{p} \omega_{p} / \omega_{0} \approx 3.2 \times 10^{-3},
$$

which yields a signal gain $G=10 \log \left(\cosh ^{2} g N\right) \approx 22 \mathrm{~dB}$ $>20 \mathrm{~dB}$. This overestimate can be explained by the fact that the number of poling periods accommodated on the full length of our transmission line is not sufficiently large, $N / m \approx 2$. Thus, obvious undulations of the growing signal that were clearly seen in our simulations (see such plots, e.g., in Fig. 2.4.2 in Ref.31), are significant on this scale. However, little increase in the signal gain in such a short transmission line is still possible at a small quasi-phase mismatch, $m \lesssim 2 \ell_{c}$ (see dashed red curve in Fig. 4(b) calculated for a tapered meander with a period of $m=420)$.

Comparison of the gains obtained for different QPM profiles (see Fig. 4(b)) shows that tapering of the profile leads to an appreciable reduction in rippling (see curves (ii) and (iii)). Although the sinusoidal profile (iii) ensures the smoothest possible frequency dependence, the gain level at a similar length $N$ and the similar pump amplitude $\phi_{p}$ is somewhat smaller (ca. $-2 \mathrm{~dB}$ ) than for the tapered meander (ii) having larger $\eta_{\text {eff. }}$. Thus, exploiting profile (ii) seems to be a good trade-off between maximum gain and smoothness of spectrum. The resulting $3 \mathrm{~dB}$ bandwidth is ca. $0.4 \omega_{p}$ (cf. with the figure for ideal $3 \mathrm{WM}$ amplifier of $\left.0.47 \omega_{p}^{25,26}\right)$. For the given circuit parameters, the signal bandwidth is about $6 \mathrm{GHz}$.

In conclusion, we applied the optical QPM concept to a parametric amplifier for traveling microwaves, designed a poled superconducting transmission line, and demonstrated its efficiency by modeling. We showed that poling in this JTWPA with 3WM can be realized using a simple architecture with periodically inverted groups of identical rf-SQUIDs in a uniform magnetic field. Modeling using CMEs showed that this JTWPA can have a relatively large gain over its wide bandwidth. Moreover, the QPM approach can also be applied to JTWPAs with 3WM based on SNAILs (Fig. 1(c)). Finally, periodic inverting of every second rf-SQUID (or SNAIL) creates a simple design of the transmission line with a fully suppressed nonlinearity of the $\chi^{(2)}$-type $(\beta=0)$ and widely tunable Kerr coefficient $\gamma \stackrel{16,17}{1}$ Such JTWPAs can be operated in pure 4WM regime with a tunable negative value of $\gamma$, which was exploited in a recent study of a JTWPA with reversed Kerr nonlinearity $\underline{\underline{8}}$ 
In summary, we believe, that by using the QPM concept in JTWPAs with 3WM, these amplifiers will become the practical devices and support their wide application in quantum technologies.

The author would like to acknowledge useful discussions with T. Dixon, R. Dolata, and C. Kißling. This work was partially funded by the Joint Research Project ParaWave of the European Metrology Programme for In- novation and Research (EMPIR). This project 17FUN10 ParaWave has received funding from the EMPIR programme co-financed by the Participating States and from the European Union Horizon 2020 research and innovation programme.

The data that support the findings of this study are openly available in Zenodo at https://doi.org/10.5281/zenodo.4732453, Ref. 38.
* Electronic address: alexander.zorin@ptb.de

1 W. H. Louisell, A. Yariv, and A. E. Siegman, Quantum fluctuations and noise in parametric processes. I, Phys. Rev. 124, 1646 (1961).

2 B. H. Eom, P. K. Day, H. G. LeDuc, and J. Zmuidzinas, A wideband, low-noise superconducting amplifier with high dynamic range, Nat. Phys. 8, 623 (2012).

3 M. R. Vissers, R. P. Erickson, H.-S. Ku, L. Vale, W. Xian, G. C. Hilton, and D. P. Pappas, Low-noise kinetic inductance traveling-wave amplifier using three-wave mixing, Appl. Phys. Lett. 108, 012601 (2016).

4 M. Malnou, M. R. Vissers, J. D. Wheeler, J. Aumentado, J. Hubmayr, J. N. Ullom, and J. Gao, A three-wave mixing kinetic inductance traveling-wave amplifier with near-quantum-limited noise performance, PRX Quantum 2, 010302 (2021).

${ }^{5}$ C. Macklin, K. O'Brien, D. Hover, M. E. Schwartz, V. Bolkhovsky, X. Zhang, W. D. Oliver, and I. Siddiqi, A near-quantum-limited Josephson traveling-wave parametric amplifier, Science 350, 307 (2015).

6 T. C. White, J. Y. Mutus, I.-C. Hoi, R. Barends, B. Campbell, Yu Chen, Z. Chen, B. Chiaro, A. Dunsworth, E. Jeffrey, et al., Traveling wave parametric amplifier with Josephson junctions using minimal resonator phase matching, Appl. Phys. Lett. 106, 242601 (2015).

7 L. Planat, A. Ranadive, R. Dassonneville, J. P. Martínez, S. Léger, C. Naud, O. Buisson, W. Hasch-Guichard, D. M. Basko, and N. Roch, Photonic-Crystal Josephson Traveling-Wave Parametric Amplifier, Phys. Rev. X 10, 021021 (2020).

8 A. Ranadive, M. Esposito, L. Planat, E. Bonet, C. Naud, O. Buisson, W. Guichard, and N. Roch, A reversed Kerr traveling wave parametric amplifier, arXiv:2101.05815

9 A. Wallraff, D. I. Schuster, A. Blais, L. Frunzio, R.-S. Huang, J. Majer, S. Kumar, S. M. Girvin, and R. J. Schoelkopf, Strong coupling of a single photon to a superconducting qubit using circuit quantum electrodynamics, Nature (London) 431, 162 (2004).

10 M. H. Devoret and R. J. Schoelkopf, Superconducting circuits for quantum information: An outlook, Science 339, 1169 (2013).

11 R. Movshovich, B. Yurke, P. G. Kaminsky, A. D. Smith, A. H. Silver, R. W. Simon, and M. V. Schneider, Observation of zero-point noise squeezing via a Josephson-parametric amplifier, Phys. Rev. Lett. 65, 1419 (1990).

12 M. A. Castellanos-Beltran and K. W. Lehnert, Widely tunable parametric amplifier based on a superconducting quantum interference device array resonator, Appl. Phys. Lett. 91, 083509 (2007).

13 T. Yamamoto, K. Inomata, M. Watanabe, K. Matsuba, T. Miyazaki, W. D. Oliver, Y. Nakamura, and J. S. Tsai,
Flux-driven Josephson parametric amplifier, Appl. Phys. Lett. 93, 042510 (2008).

14 G. P. Agrawal, Nonlinear fiber optics (Academic press, San Diego, California, 2007).

15 K. O'Brien, C. Macklin, I. Siddiqi, and X. Zhang, Resonant phase matching of Josephson junction traveling wave parametric amplifiers, Phys. Rev. Lett. 113, 157001 (2014).

16 M. T. Bell and A. Samolov, Traveling-wave parametric amplifier based on a chain of coupled asymmetric SQUIDs, Phys. Rev. Applied 4, 024014 (2015).

17 W. Zhang, W. Huang, M. E. Gershenson, and M. T. Bell, Josephson metamaterial with a widely tunable positive or negative Kerr constant, Phys. Rev. Applied 8, 051001 (2017).

18 H. R. Mohebbi and A. H. Majedi, Analysis of seriesconnected discrete Josephson transmission line, IEEE Trans. Microwave Theory Tech. 57, 1865 (2009).

19 O. Yaakobi, L. Friedland, C. Macklin, and I. Siddiqi, Parametric amplification in Josephson junction embedded transmission lines, Phys. Rev. B 87, 144301 (2013).

20 A. B. Zorin, Josephson traveling-wave parametric amplifier with three-wave mixing, Phys. Rev. Applied 6, 034006 (2016).

21 N. E. Frattini, U. Vool, S. Shankar, A. Narla, K. M. Sliwa, and M. H. Devoret, 3-wave mixing Josephson dipole element, Appl. Phys. Lett. 110, 222603 (2017).

22 A. B. Zorin, M. Khabipov, J. Dietel, and R. Dolata, Traveling-wave parametric amplifier based on three-wave mixing in a Josephson metamaterial, 2017 16th International Superconductive Electronics Conference (ISEC), Naples, 2017, pp. 1-3, doi: 10.1109/ISEC.2017.8314196.

23 A. Miano and O. A. Mukhanov, Symmetric traveling wave parametric amplifier, IEEE Trans. Appl. Supercond. 29, 1501706 (2019).

24 A. B. Zorin, Flux-driven Josephson traveling-wave parametric amplifier, Phys. Rev. Applied 12, 044051 (2019).

25 P. K. Tien, Parametric amplification and frequency mixing in propagating circuits, J. Appl. Phys. 29, 1347 (1958).

26 A. L. Cullen, Theory of the traveling-wave parametric amplifier, Proc. IEE - Part B: Electron. and Communication Eng. 107, 101 (1960).

27 R. P. Erickson and D. P. Pappas, Theory of multiwave mixing within the superconducting kinetic-inductance traveling-wave amplifier, Phys. Rev. B 95, 104506 (2017).

28 T. Dixon, J. W. Dunstan, G. B. Long, J. M. Williams, P. J. Meeson, and C. D. Shelly, Capturing complex behaviour in Josephson travelling wave parametric amplifiers, Phys. Rev. Applied 14, 034058 (2020).

29 J. M. Manley and H. E. Rowe, Some general properties of nonlinear elements, part 1. General energy relations, Proc. IRE 44, 904 (1956). 
30 J. A. Armstrong, N. Bloembergen, J. Ducuing, and P. S. Pershan, Interactions between light waves in a nonlinear dielectric, Phys. Rev. 127, 1918 (1962).

31 R. W. Boyd, Nonlinear optics (Academic Press, London, 2008).

32 M. Charbonneau-Lefort, B. Afeyan, and M. M. Fejer, Optical parametric amplifiers using chirped quasiphasematching gratings I: practical design formulas, J. Opt. Soc. Am. B 25, 463 (2008).

${ }^{33}$ R. L. Byer, Quasi-phasematched nonlinear interactions and devices, J. Nonlinear Opt. Phys. Mater. 6, 549 (1997).

${ }^{34}$ M. M. Fejer, G. A. Magel, D. H. Jundt, R. L. Byer, Quasiphase-matched second harmonic generation: tuning and tolerance, IEEE J. Quantum Electron. 28, 2631 (1992).

35 M. Yamada, N. Nada, M. Saitoh, and K. Watanabe, Firstorder quasi-phase matched $\mathrm{LiNbO}_{3}$ waveguide periodically poled by applying an external field for efficient blue secondharmonic generation, Appl. Phys. Lett. 62, 435 (1993).

${ }^{36}$ M. Fiorentino, S. M. Spillane, R. G. Beausoleil, T. D. Roberts, P. Battle, and M. W. Munro, Spontaneous parametric down-conversion in periodically poled KTP waveguides and bulk crystals, Opt. Express 15, 7479 (2007).

37 K. K. Likharev, Dynamics of Josephson junctions and circuits (Gordon and Breach, New York, 1986).

38 A. B. Zorin, Quasi-phasematching in a Josephson traveling-wave parametric amplifier. Additional information, https://doi.org/10.5281/zenodo.4732453

39 J. Huang, X. P. Xie, C. Langrock, R. V. Roussev, D. S. Hum, and M. M. Fejer, Amplitude modulation and apodization of quasiphase-matched interactions, Opt. Lett. 31, 604 (2006). 